\title{
Application of Analog-Circuit Diagnosis Method to Fault Location in Distribution Networks
}

\author{
Minfang Peng, Meie Shen, Jianbiao He, Liang Zhu, and Hongwei Che
}

\begin{abstract}
Analog circuit fault diagnosis method is introduced to line fault location in a distribution network. The line fault location based on analog circuit diagnosis theory can be performed by fault line sector identification and distance estimation followed. This paper deals mainly with fault line sector identification. A new technique depending on obtaining measurable nodes voltage response corresponding to a particular current excitation at the header terminal of distribution lines is proposed. Line fault sector is determined by the proposed fault detection criterion.
\end{abstract}

Index Terms-Fault diagnosis, fault line sector identification, analog circuit, distribution network.

\section{INTRODUCTION}

The improvement of the quality of electricity distribution is actually a subject of increasing interest. Fast and accurate line fault location in distribution networks is essential to improve the quality of supply, which is one basic requirement of modern society. However, the traditional practice of manual switching and circumambulating consumes a lot of time. After working hard for several years, many grounding line selection protections and fault location approaches are developed [1]-[4]. But up to now, the accuracy of fault location is unsatisfactory. Thus, to search for an effective fault location method for the distribution system in order to locate the actual feeder faults becomes more and more important.

This paper applies analog circuit diagnosis method to line fault location for a distribution network. In the following part of this paper, the appropriate theory of analog fault diagnosis is briefly introduced. Then the possibility of locating feeder faults by analog fault diagnosis method is expounded. A new technique of feeder fault detection based on analog fault diagnosis theory is presented, which performs fault location through two steps including fault line sector identification and distance estimation. The method for fault line sector

Manuscript received March 9, 2013; revised June 28, 2013. This work was supported by National Natural Science Foundation of China under Grants 61173108, 61272147, 60973032 and 60673084, and Hunan Provincial Natural Science Foundation of China under Grants 06JJ4075 and $10 \mathrm{JJ} 2045$

Minfang Peng is with the College of Electrical and Information Engineering, Hunan University, Changsha, 410082, China (e-mail: pengminfang@ hnu.edu.cn).

Meie Shen is with the College of Computer Science, Beijing University of Information Science and Technology, Beijing,100101, China (e-mail: smelk@163.com)

Jianbiao He is with the School of Information Science \& Engineering, Central South Uinversity, Changsha, 410083, China, (e-mail: jbhe@ mail.csu.edu.cn)

Liang Zhu and Hongwei Che are with the Hunan province Electric Power Company, China identification is discussed in detail. By obtaining measurable nodes voltage response corresponding to a particular current excitation at the header terminal of distribution lines, the fault line sector is determined by means of a fault detection criterion including the voltage responses. The feasibility of the method is then verified by the results of simulation for faulted distribution lines.

\section{ANAlOG-CiRCUIT FAUlt DiagnOSIS PRINCIPLE}

Analog circuit fault diagnosis includes detecting faulty circuits, locating or identifying faulty components, and determining their parameter ranges where faults occur. In recent years, attention has been directed to fault detection and location. A number of approaches have been proposed to address this problem. $\mathrm{K}$-fault diagnosis method is a typical analog fault diagnosis approach. The theoretical basis for $\mathrm{k}$-fault diagnosis is derived under the assumption that there are $\mathrm{k}$ simultaneous faults within the diagnosed network and the affection of a fault can be replaced by an appropriate current source that is called fault current. Thus, fault detection can be performed through checking the fault current. Obviously, there is no fault if all of the fault current sources are zero, and there is a corresponding fault if any of fault current sources is not zero. Usually, $\mathrm{k}$-fault diagnosis is classified into k-node-fault diagnosis and k-branch-fault diagnosis.

K-node-fault diagnosis [5]-[7] can be done by checking node fault current in order to identify faulty nodes possessing fault current and then locating faulty branches (or elements) according to fault current.

Assume that the network $N$ under consideration consists of $n$ nodes and $b$ branches. Assume again that node $0,1,2, \ldots, m$ among the nodes are accessible nodes.

The normal network is described by

$$
Y_{n} V_{n}=I_{n}
$$

where $Y_{n}$ is nodes admittance matrix. $V_{n}$ and $I_{n}$ represent nodes voltage vector and nodes current excitation vector respectively.

Suppose that $Y_{n}$ becomes $Y_{n}+\Delta Y_{n}$ because of faults. When the faulted network has the same nodes current excitation as the previous normal network, we have

$$
\left(Y_{n}+\Delta Y_{n}\right)\left(V_{n}+\Delta V_{n}\right)=I_{n}
$$

where $\Delta V_{n}$ is nodes voltage change vector.

According to (1) and (2), we have 


$$
Y_{n} \times \Delta V_{n}+\Delta Y_{n}\left(V_{n}+\Delta V_{n}\right)=0
$$

That is

$$
\Delta V_{n}=Z_{n} J_{n}
$$

here $Z_{n}=Y_{n}^{-1}$

$$
J_{n}=-\Delta Y_{n}\left(V_{n}+\Delta V_{n}\right)
$$

where $J_{n}$ is nodes fault current vector

Considering that current excitation and voltage measurement are performed only at the $\mathrm{m}$ accessible ports, we have

$$
I_{n}=\left[\begin{array}{c}
I_{m} \\
0
\end{array}\right]
$$

Thus, (3) becomes

$$
\Delta V_{m}=Z_{m m} J_{n}
$$

where $Z_{m m}$ is ports impedance matrix corresponding to the accessible ports.

Equation (5) is known as k-node-fault diagnosis equation. If the condition on k-node-fault testability is satisfied, the faulty node set in a network is determined by checking the non-zero element among fault current vector $J_{n}$ at equation (5).

K-branch-fault diagnosis [7], [8] can be done by checking branch fault current in order to locate faulty branches possessing fault current and then valuing faulty branches by means of multiple-excitation method. K-branch-fault location equation can be obtained from (5). From (5) and

$$
J_{n}=A J_{b}
$$

here $A$ is incidence matrix and $J_{b}$ is branches fault current vector, we have

$$
\Delta V_{m}=Z_{m b} J_{b}
$$

where

$$
\begin{aligned}
& J_{b}=-\Delta Y_{b} A^{T}\left(V_{n}+\Delta V_{n}\right)=-\Delta Y_{b}\left(V_{b}+\Delta V_{b}\right) \\
& Z_{m b}=A Z_{m m}
\end{aligned}
$$

here $Z_{m b}$ is transfer impedance matrix.

Equation (7) is known as k-branch-fault diagnosis equation. If the condition on k-branch fault testability is satisfied, the faulty branch set in a network is determined by checking the non-zero element among fault current vector $J_{b}$ at (5).

This task can be fulfiled by the following proposition. [Proposition ] Let

$$
T_{i}=\left[\begin{array}{lllll}
Z_{i 1} & Z_{i 2} & \cdots & Z_{i k} & \Delta V_{m}
\end{array}\right]
$$

where $Z_{i 1} \quad Z_{i 2} \quad \cdots \quad Z_{i k}$ are any k column vectors of $Z_{m b}$.

Consume that the condition on $\mathrm{k}$-branch fault testability is satisfied. If

$$
\operatorname{rank}\left(T_{j}\right)=k
$$

there are not more than $k$ faulty branches in the network, and the faulty branches is certainly included by the $k$ branches corresponding to $T_{j}$.

\section{FAUlT LOCATION FOR DiSTRIBUTION NETWORKS}

Generally, since there is only one faulty line sector possible among distribution network, we have $k \leq 3$ corresponding to $\mathrm{k}$-branch-fault diagnosis and $k \leq 4$ to k-node-fault diagnosis. Particularly, most of line faults are single-phase faults. Thus we have $k=1$ and $k=2$ corresponding to k-branch-fault diagnosis and k-node-fault diagnosis respectively. On the other hand, with the improvement of distribution automation, voltage measurement at the main nodes of distribution network and information transmission among them has become possible. Thus, the condition on k-branch fault testability can be satisfied for distribution lines, and any of $\mathrm{k}$-fault diagnosis methods can be used to locate line faults in distribution network.

Considering the difficulty that k-node-fault diagnosis can be performed only when all accessible nodes are excited by current signal, we choose k-branch-fault diagnosis for line fault location. It can be done when the header terminal of distribution lines is excited by particular current signal. According to the theory of k-branch-fault diagnosis mentioned above, line fault location should be divided into two stages, i.e., fault line sector identification corresponding to fault branch location of k-branch-fault diagnosis and distance estimation to parameter valuation of fault branches.

In order to locate line faults, an appropriate circuit should be modeled after the distribution network at first. Every line sector is modeled by a $\Pi$ equivalent circuit shown as Fig. 1. The parameters $x$ and $b$ of the circuit are calculated according to the situation that header terminal of single phase of lines is excited by the current source with much higher frequency than industrial frequency. Parameters $g$ is approximately zero. Similarly, transformers can be modeled by their relevant equivalent circuits as Fig. 2 and Fig. 3.

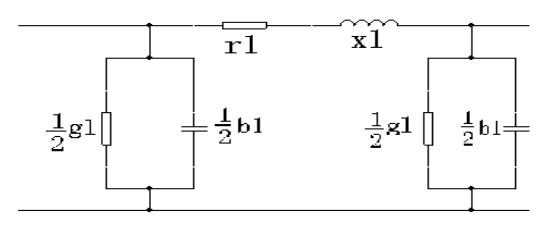

Fig. 1. П equivalent circuit of a line sector.

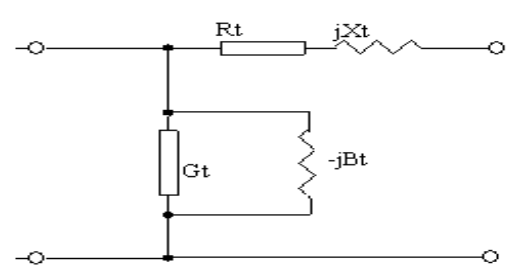

Fig. 2. Equivalent circuit of a transformer with 2 windings. 


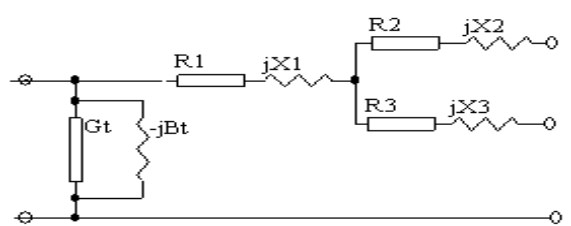

Fig. 3. Equivalent circuit of a transformer with 3 windings.

Then, select some appropriate measurable nodes so as to satisfy the condition on k-branch fault testability. Thus, the transfer impedance matrix $Z_{m b}$ of distribution lines and the accessible nodes voltage matrix $V_{m}$ in normal network is calculated.

When line fault occurs, the header terminal of every phase of lines is excited for a time by an independent current signal with much higher frequency than industrial frequency and the corresponding voltage matrix $V_{m}^{f}$ of accessible nodes is measured. Thus, we can obtain $\Delta V_{m}$ that is

$$
\Delta V_{m}=V_{m}^{f}-V_{m}
$$

According to the proposition introduced in part $\Pi$, in order to evaluate the actual state of the network and detect fault line sector, it is necessary to search for $j$ which makes

$$
\operatorname{rank}\left(T_{j}\right)=k
$$

Thus, $k$ is number of faulted phases and $T_{j}$ includes the faulted line sector as well as the faulted line. The method mentioned above can be expressed in the flow diagrams as (a) and (b) in Fig. 4.

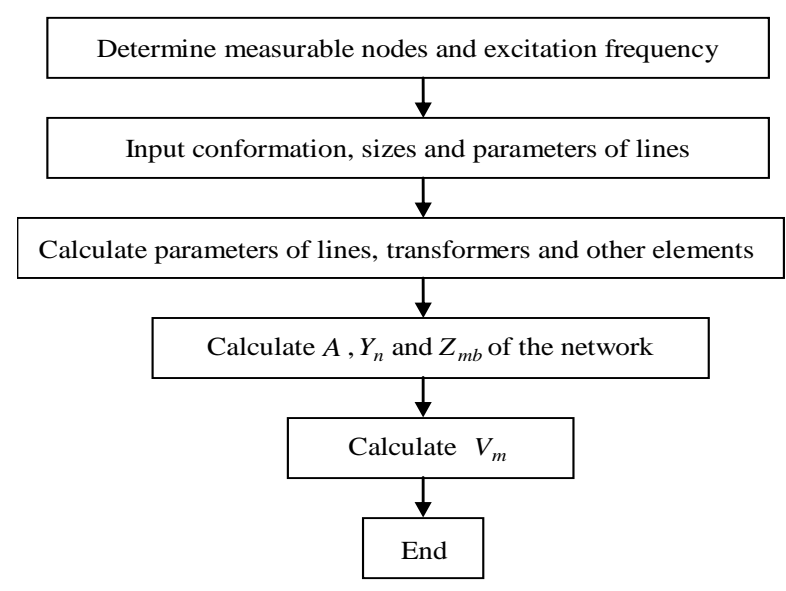

(a) Simulation before test

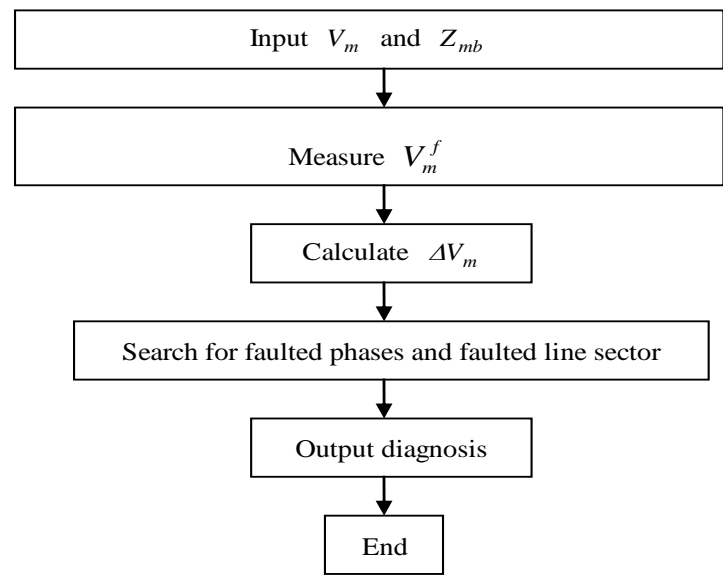

(b) Diagnosis after test

Fig. 4. Process of fault line sector identification.
After determining the faulted line sector, parameters of the faulty line can be calculated by means of multi-excitation k-branch-fault diagnosis method [5]. Thus, the fault distance can be estimated according to the parameters.

\section{AN EXAMPLE FOR LINE FAULT LOCATION}

The distribution line shown as Fig.4 is a simplified model of an actual distribution network. The frequency of current excitation for measure is $1 \mathrm{kHz}$. The nodes of the network are measurable nodes.

Parameters of the lines is: $\mathrm{L}_{1}=8 \mathrm{~km}, \mathrm{~L}_{2}=12 \mathrm{~km}, \mathrm{~L}_{3}=10 \mathrm{~km}$, $\mathrm{L}_{4}=15 \mathrm{~km}, \quad \mathrm{~L}_{5}=7 \mathrm{~km}, \quad \mathrm{~L}_{6}=5 \mathrm{~km}, \quad \mathrm{~L}_{7}=8 \mathrm{~km}, \quad \mathrm{~L}_{8}=10 \mathrm{~km}$, $r=0.18 \Omega / \mathrm{km}, x=10.24 \Omega / \mathrm{km}, g=0, b=3.86 \times 10-5 \mathrm{~s} / \mathrm{km}$.

According to the method mentioned above, various kinds of line faults including single-phase short-circuit, single-phase open-circuit faults and two-phase short- circuit faults occurring at $\mathrm{L}_{1} \sim \mathrm{L}_{8}$ respectively are simulated and accurate results of corresponding fault line sector identification have been obtained.

Notes that there is the deviation of counting parameters of lines and transformers from the actual parameters as well as the error of measurement and calculation parameters of lines and transformers. Thus, accurate results may not be obtained by means of the fault detection criterion (8). Some effective methods described in References [9]-[16] may be used to solve this problem.

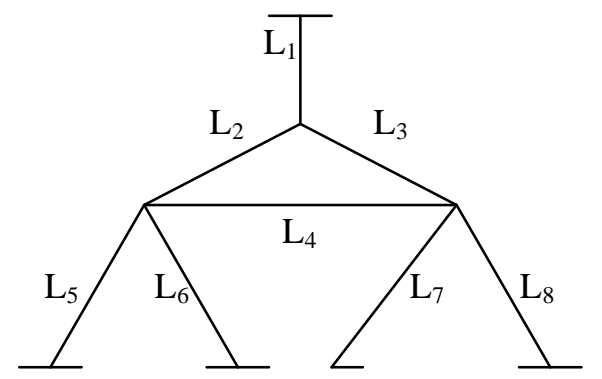

Fig. 4. Simplified model of an actual distribution lines.

\section{CONCLUSION}

A new approach to line fault location in distribution networks has been presented which is based upon k-fault diagnosis. It performs line fault location by two steps, i.e., fault line sector identification and distance estimation. The theory is applicable to single-phase fault as well as multiple-phase fault location and depends upon measuring measurable nodes voltages due to current excitations applied at header terminal of distribution lines. A criterion of line fault sector detection is proposed. The feasibility of the theory has been verified by the results of simulation for faulted distribution lines.

An extensive search of the literature reveals that the approach proposed in this paper is conceptually different from all known methods of line fault location in distribution networks. Further detailed research is now required in order to further refine the method. The early results presented have indicated it is worthwhile performing such further work.

Researches are now required to determine fault line sector detection criterion when there is the deviation of counting parameters of lines and transformers from the actual 
parameters as well as the error of measurement and calculation parameters of lines and transformers. Researches on the detailed method of distance estimation are also being undertaken.

\section{REFERENCES}

[1] S. M. Brahma, "Fault location in power distribution system with penetration of distributed generation," IEEE Trans. Power Del., vol. 26 no. 3, pp. 1545-1553, 2011.

[2] M. J. B. Reddy and D. K. Mohanta, "Performance evaluation of an adaptive-network-based fuzzy inference system approach for location of faults on transmission lines using monte carlo simulation," IEEE Trans Fuzzy sys, vol. 16, no. 4, pp. 909-919, 2008.

[3] M. Pourahmadi-Nakhli and A. A. Safavi, "Path characteristic frequency-based fault locating in radial distribution systems using wavelets and neural networks," IEEE Trans. Power Del, vol. 26, no. 2 , pp. 772-781, 2011

[4] V. Calderaro, A. Piccolo, and V. Galdi et al., "Identifying fault location in distribution systems with high distributed generation penetration," IEEE Africon, pp. 1-6, 2009

[5] Z. F. Huang, C. S. Lin, and R. W. Liu, "Node fault diagnosis and design of testability," IEEE Trans. On CAS, vol. CAS-30, pp. 257, 1983.

[6] J. W. Bandler and A. E. Salama, "Fault diagnosis of analog circuits," in Proc. IEEE, vol. 73, no. 8, pp. 1279, 1985.

[7] S. Y. Yang, Fault Diagnosis of Analog Circuits and Design of Testability, Tsinghua University Publishing House, 2001.

[8] R. M. Biernachi and J. W. Bandler, "Multi-fault location of analog circuits," IEEE Trans. On CAS, vol. CAS-28, no. 5, pp. 361, 1981.

[9] R. Zou and J. M. Huang, "Fault location of linear nonreciprocal circuit with tolerance," in Proc. IEEE Int. Symp., pp. 1163, 1988

[10] L. Hu, Z. F. Huang, Y. F. Huang, and R. Liu, "A stochastic model fault diagnosis with tolerance," IEEE Int. Symp. Circuits Syst., pp. 680 , 1984.

[11] Z. F. Huang and R. Liu, "Analog fault diagnosis with tolerance," IEEE Int. Symp. Circuits Syst., pp. 1332, 1986.

[12] Y. Togawa, T. Matsumoto, and H. Arai, "Linear algorithm for branch fault diagnossis of analog circuits: TF equivalence-class approach," IEEE Trans. on Circuits Syst., vol. CAS-33, no. 10, pp. 992, 1986.

[13] L. C. Suen and R. Liu, "Determination of structure of multivariable stochatic linear system," IEEE Trans. on Autom. Control, vol. AC-23, no. 3 , pp. 458, 1978.

[14] M. F. Peng and Y. G. He, "Optimum fault screening location of Analog circuits with tolerance," presented at IEEE 6th Conf. SCI, Orlando, USA, July $14-18,2002$.

[15] M. F. Peng and Y. G. HE, "Study on k-fault screen method in circuits with tolerance," Journal of Circuits and Systems, vol. 7, no. 2, pp. 92, 2002.

[16] M. F. Peng and Y. G. HE, "Algorithm for k-fault fuzzy screen diagnosis in analog circuits with tolerance," Microelectronics and Computer, vol. 19, no. 2, pp. 40, 2002.

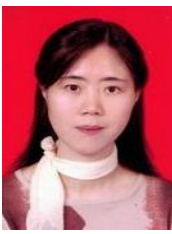

processing.

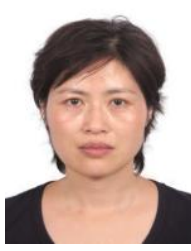

processing.
Min-fang Peng was born in Hunan, China, in 1964. She received the Ms. degree in 1992 and $\mathrm{Ph}$. D. degree in 2006 , both in electrician theory from Hunan University, China.

She works as a professor at the college of electrical and information engineering in Hunan University. Her fields of interest are in test and diagnosis of analog circuits, fault diagnosis of power system, intelligent information

Meie Shen was born in Jiangsu, China, in 1966. She received the Ms. degree in 1989 in 2006, in automation from Huazhong University of Science and Technology, China.

She works as an associate professor in the Beijing University of Information Science and Technology. Her fields of interest are in test and diagnosis of analog circuits, computer simulation and intelligent information 